\title{
CORRECTIONS
}

\section{Removing the hype from hypertension}

In the competing interests section of this Editorial ( $B M J$ 2014;348:g1937, doi:10.1136/bmj.g1937), the second NCT number that the authors listed for a trial they conducted is incorrect. This number should have read NCT01639378, not "NCT0163938."
Cite this as: BMJ 2014;348:g2088

๑ BMJ Publishing Group Ltd 2014 